LUBMAN, D. I., VELAKOULIS, D., McGORRY, P. D., et al (2002) Incidenta radiological findings on brain magnetic resonance imaging in first-episode psychosis and chronic schizophrenia. Acta Psychiatrica Scandinavica, 106, 331-336.

LUKOFF, D., LIBERMAN, R. P. \& NUECHTERLEIN, K. H. (1986) Symptom monitoring in the rehabilitation of schizophrenic patients. Schizophrenia Bulletin, 12, 578-602.

MALLA, A. K. \& NORMAN, R. M. G. (2002) Early intervention in schizophrenia and related disorders: advantages and pitfalls. Current Opinion in Psychiatry, 15, 17-23.

MCGORRY, P. D., SINGH, B. S. COPOLOV, D. L., et al (1990) Royal Park Multi-Diagnostic Instrument for Psychosis: II. Development, reliability, and validity. Schizophrenia Bulletin, 16, 517-536.

McGORRY, P., KILLACKEY, E., ELKINS, K., et al (2003) SummaryAustralian and New Zealand clinical practice guideline for the treatment of schizophrenia. Australasian Psychiatry 11, 136-147.

NATIONAL COLLABORATING CENTRE FOR MENTAL HEALTH (2003) Schizophrenia. Full National Clinical

Guidance on Core Interventions in Primary and Secondary Care. London \& Leicester: Gaskell \& British Psychological Society.

POWER, P., ELKINS, K., ADLARD, S., et al (1998) Analysis of the initial treatment phase in first-episode psychosis. British Journal of Psychiatry, 172 (suppl. 33), 71-76.
SCHWARTZ, J.E., FENNIG, S.,

WADE, D., HARRIGAN, S., McGORRY, TANENBERG-KARANT, M et al (2000) Congruence of diagnoses 2 years after a first-admission diagnosis of psychosis. Archives of General Psychiatry, 57, 593-600. $P$, et al (2004) The impact of substance use disorders on recovery and relapse rates, and service use, in first-episode psychosis. Schizophrenia Research, 67 (suppl. 1), 221.

SIPOS, A., HARRISON, G., GUNNELL, D., YUNG, A. R., ORGAN, B. A. \& HARRIS, et al (2001) Patterns and predictors of M. G. (2003) Management of early hospitalisation in first-episode psychosis in a generic adult mental psychosis. Prospective cohort study. health service. Australian and New British Journal of Psychiatry, 178, Z Zealand Journal of Psychiatry, 37. 518-523. 429-436.

*Darryl Wade Clinical Psychologist, ORYGEN Youth Health, Locked Bag 10, Parkville 3052, Australia, e-mail: darryl.wade@mh.org.au, Susy Harrigan Research Fellow, ORYGEN Research Centre, University of Melbourne and Senior Research Officer, School of Population Health, University of Queensland, Meredith G. Harris Research Fellow, Department of Psychiatry, University of Melbourne, Jane Edwards Clinical Director, ORYGEN Youth Health and Department of Psychiatry, University of Melbourne, Patrick D. McGorry Director ORYGEN Youth Health, Executive Director, ORYGEN Research Centre and Professor, Department of Psychiatry, University of Melbourne, Australia

\title{
Has the referral of older adults with dementia changed since the availability of acetylcholinesterase inhibitors and the NICE guidelines?
}

\section{AIMS AND METHOD}

To investigate whether patients with dementia are referred to specialist services earlier in the disease since the launch of acetylcholinesterase inhibitors and the publication of the National Institute for Clinical Excellence (NICE) guidelines for the use of these drugs. All referrals to

\author{
old age psychiatry services in two \\ 6-month periods in 1996 and 2003 \\ were surveyed retrospectively for \\ diagnosis, Mini-Mental State \\ Examination (MMSE) score and use of \\ acetylcholinesterase inhibitors.

RESULTS
The mean MMSE score at referral
increased from 18.8 to 21.5
$(P=0.0005)$ between 1996 and 2003 .

Acetylcholinesterase inhibitors were prescribed for $35 \%$ of all patients and $58 \%$ of patients that would be suitable according to NICE guidelines in the 2003 group.

\section{CLINICAL IMPLICATIONS}

The earlier referral of patients with dementia to mental health services is encouraging.
Donepezil was launched in the UK by Pfizer in March 1997 as the first readily available pharmacological treatment to slow the rate of cognitive decline in Alzheimer's disease Donepezil increases the available acetylcholine by inhibition of the enzyme acetylcholinesterase (AChE). This was followed in 1998 by the launch of rivastigmine by Novartis and in 2000 by galantamine from Shire Pharmaceuticals. There is also evidence that these drugs may have some benefit in the cognitive decline associated with cerebrovascular dementia (Malouf \& Birks, 2004) and behavioural disturbance in Lewy body dementia (McKeith et al, 2000).

About the time of the launch of these drugs there were a number of initiatives designed to improve the care of older adults with mental health problems. These included the Forget Me Not reports (Audit Commission, 2000, 2002) and the National Service Framework for Older People (Department of Health, 2001). Arguably, however, it was the review of the AChE inhibitors by the National Institute for Clinical Excellence (NICE) in January 2001 that brought a new mood of optimism in the diagnosis and management of dementia (O'Brien \& Ballard, 2001). NICE recommended that the three drugs should be available for National Health Service (NHS) patients with mild or moderate Alzheimer's disease, whose Mini-Mental State Examination (MMSE; Folstein et al, 1975) score is above 12 , with an assessment of effec- 
tiveness after 2-4 months. The value of these drugs in other forms of dementia was not assessed.

The advent of treatments that are both effective

original papers and available on the NHS for early Alzheimer's disease should have led to a profound alteration in patterns of referral from primary care to specialist services for patients with dementia to allow diagnosis and initiation of treatment early in their illness. This effect has not, to our knowledge, ever been studied. In addition, it is possible that the proportion of dementias diagnosed as Alzheimer's, vascular, Lewy body, etc. may have altered since the publication of the NICE guidelines, either because of a different patient population being referred or to maximise the use of the AChE inhibitors. The impact of the NICE recommendations on healthcare provision has recently been brought into question (Sheldon et al, 2004; White, 2004), and the efficacy of AChE inhibitors continues to be debated (AD2000 Collaborative Group, 2004).

Old age psychiatry services in the West Suffolk area cover a mixed urban and rural population with approximately 42000 people aged 65 years and over. This study examines the effect the availability of the AChE inhibitors and the publication of the NICE guidelines have had on referrals to old age psychiatrists in the West Suffolk region from primary care, examining the MMSE score on referral, diagnosis and the use of AChE inhibitors. The primary null hypothesis is that the MMSE scores at referral have not changed between 1996 and 2003.

\section{Method}

We retrospectively examined records of all patients referred from primary care to specialist old age psychiatry services in West Suffolk during two 6-month periods from July to December 1996, before AChE inhibitors were available, and the same months in 2003. The details of all patients referred from primary care are kept centrally and subsequent records are computerised. We looked at the diagnoses for all new referrals following the patient's first appointment and collected data from the record of this first appointment if the diagnosis included cognitive impairment thought to be a result of any form of dementia. This would include those patients diagnosed with mild cognitive impairment, although this was not used clinically.
For this study, we obtained patients' age and gender, recorded diagnosis, MMSE score if measured and whether an AChE inhibitor was prescribed at the first consultation. If the MMSE score was out of less than 30 (owing, for example, to poor eyesight) the score was converted to an equivalent score out of 30. The MMSE scores in the two samples were compared with a twotailed Student's $t$-test, and the diagnoses compared with a $\chi^{2}$ test.

\section{Results}

In the first sample (6 months in 1996), 237 new patients were referred to the old age psychiatry service. Of these, 7 did not see an old age psychiatrist (3 died, 1 was admitted to the acute hospital, 3 records were not available) and of the remaining 230, 112 had a new diagnosis of dementia at referral (40 men (36\%) and 72 women $(64 \%))$. The mean age was 81.7 years, with a range of 66-96 years. The MMSE scores were available for 86 patients (77\%).

In the second sample (6 months in 2003), 275 new patients were referred. Of these, 19 did not see an old age psychiatrist ( 2 died, 3 were admitted to the acute hospital, 4 were seen in a different area, 7 were seen by other professionals and no records were available for 3 ). Of the remaining 256 patients, 148 had a new diagnosis of dementia ( 62 men (42\%) and 86 women (58\%)). The mean age of the second sample was 81.6 years with a range of 60-97 years. The MMSE scores were available for 133 patients (90\%).

The MMSE scores increased from a mean score of 18.8 (range $2-27$, s.d. $=6.32$ ) in 1996 to a mean score of 21.5 (range $8-29$, s.d. $=4.95)$ in 2003 ( $P=0.0005$; see Fig. 1). The diagnoses in the two samples are recorded in Table 1 and show considerable similarity between the two samples, with Alzheimer's disease as the most common ( $55 \%$ and $49 \%$, NS) followed by vascular dementia ( $26 \%$ and 30\%, NS) and mixed dementia.

A total of 52 of 148 patients with a new diagnosis of dementia (2003 sample) were prescribed an AChE inhibitor at the first consultation, 45 donepezil (30\%), 5 rivastigmine (3.4\%) and 2 galantamine (1.4\%); 96 patients were not prescribed these medications $(65 \%)$. There were 51 recorded MMSE scores in those patients prescribed AChE inhibitors with a mean of 23.1 (s.d. $=3.76$ ), and 82

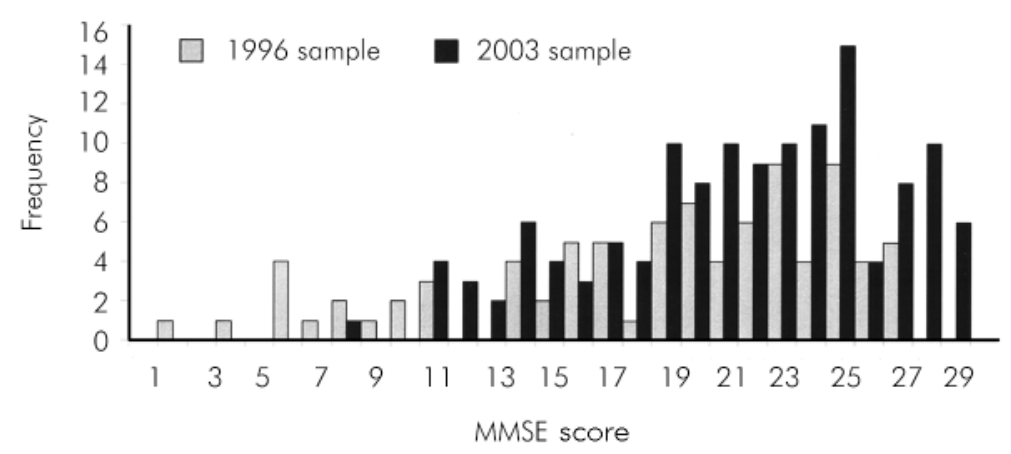

Fig. 1. Frequency of Mini-Mental State Examination (MMSE) scores in the 1996 and 2003 samples 
Table 1. Diagnoses in the 1996 and 2003 samples

\begin{tabular}{|c|c|c|}
\hline Diagnosis* & $\begin{array}{c}1996 \text { sample } \\
n(\%)\end{array}$ & $\begin{array}{c}2003 \text { sample } \\
n(\%)\end{array}$ \\
\hline Alzheimer's disease & $62(55)$ & $73(49)$ \\
\hline Vascular dementia & $29(26)$ & $45(30)$ \\
\hline Mixed dementia & $5 \quad(4.5)$ & $6(4.1)$ \\
\hline Lewy body dementia & $5 \quad(4.5)$ & $5 \quad(3.4)$ \\
\hline Frontotemporal dementia & $1 \quad(0.89)$ & $2(1.4)$ \\
\hline Unknown & $10 \quad(8.9)$ & $17(11.5)$ \\
\hline Total & 112 & 148 \\
\hline
\end{tabular}

*No statistically significant difference between 1996 and 2003 for any diagnosis.

recorded MMSE scores in patients not prescribed these drugs, with a lower mean of 20.6 (s.d. $=5.36 ; P=0.004$ ).

AChE inhibitors were prescribed for 43 patients with Alzheimer's disease (61.4\% of diagnostic group), 4 patients with vascular dementia (8.9\%), 3 patients with Lewy body dementia (60\%), 2 patients with an unknown diagnosis (11.7\%) but no patient with mixed dementia or frontotemporal dementia.

Of those patients to whom the NICE guidelines would apply (a diagnosis of Alzheimer's dementia and a MMSE score above 12, total number=72), $42(58 \%)$ received a prescription for an $\mathrm{AChE}$ inhibitor after the first consultation.

\section{Discussion}

There has been a consistent campaign through the Audit Commission's reports and the National Service Framework for Older People for the earlier diagnosis and treatment of dementia, a key aim if the NICE recommendations for the use of AChE are to be implemented. This study is the first to examine the changing pattern of referrals from primary care to specialist services since the NICE guidelines were published in 2001 and confirms that patients are being referred for specialist treatment earlier in the course of the disease.

Overall, the number of new referrals of patients with dementia rose between 1996 and 2003 from 112 to 148 , a rise of approximately $30 \%$. It is beyond the scope of this paper to examine the longer term trends in referring to specialist services, although clearly there are resource implications for the service if this referral rate continues to rise. The MMSE is a widely used guide to the severity of cognitive impairment in dementia and there was a strongly statistically significant rise in the MMSE score between the two samples, from 18.8 to 21.5 . The study included all patients whose assessment led to a diagnosis of cognitive impairment secondary to a process of dementia, and the wide range of MMSE scores in both samples shows that the diagnosis is based on factors other than simply the MMSE score itself.

The number of patients seen initially by a healthcare professional other than a doctor, such as a community psychiatric nurse, increased between 1996 and 2003 and could be a source of bias if these patients had more severe dementia. It was usual practice, however, in this area for patients without a formal diagnosis of dementia to see a medical professional initially for diagnosis and, when available, consideration of medication. The service model remained identical between the sample periods, with a slight expansion in consultant numbers that we consider unlikely to substantially influence referral patterns.

More patients in 2003 had their cognitive function assessed formally with the MMSE than in 1996, which is likely to be in part a response to the need to assess cognitive decline more accurately to determine the effectiveness of medication. Reasons for not using the MMSE were not recorded but would include failure of patients to cooperate with testing, severe dementia and prominent language skill deficits. The different use of the MMSE in the two samples would therefore tend to decrease any difference found through an apparent raising of the mean MMSE scores in 1996.

The mean age at referral remained similar between 1996 and 2003, as did the proportion of different diagnoses causing dementia. This suggests that there has not been a change in the way patients are diagnosed in order to facilitate the use of the AChE inhibitors.

The patients who received AChE inhibitors had a higher mean MMSE score than those in the 2003 sample who did not, although the mean MMSE score was still 20.6 in those not prescribed AChE inhibitors, which is well within the current NICE guidelines for patients with Alzheimer's disease. Reasons for non-prescription were not examined formally but included patient preference, contraindications and further investigations. Overall, 58\% of patients who would have been eligible for AChE inhibitors received them at first consultation.

This study shows that between 1996 and 2003 more people with suspected dementia were referred to specialist services and diagnosed earlier in their illness. A study like this is unable to directly attribute the cause of this change to NICE guidelines or associated initiatives, but it is clearly encouraging to specialists in this field that patients are seen earlier when treatments may have significant benefit to their lives.

\section{Declaration of interest}

None.

\section{References}

AD2000 COLLABORATIVE GROUP (2004) Long-term donepezil treatment in 565 patients with Alzheimer's disease (AD2000): randomised double-blind trial. Lancet, $\mathbf{3 6 3}$, $2105-2115$

AUDIT COMMISSION (2000) Forget Me Not. Mental Health Services for Older People. London: Audit Commission.

AUDIT COMMISSION (2002) Forget Me Not, Developing Mental Health
Services for Older People in England London: Audit Commission http:// www.audit-commission.gov.uk/ Products/NATIONAL-REPORT/ 3DFEF403-038C-464f-8518441477E92B15/forgetupdate.pdf

DEPARTMENT OF HEALTH (2001) National Service Framework for Older People. London: Department of Health.

FOLSTEIN, M. F., FOLSTEIN, S. E. \& MCHUGH, P. R. (1975) "Mini-menta original papers 
state". A practical method for grading the cognitive state of patients for the clinician. Journal of Psychiatric Research, 12, 189-198.

original papers
MALOUF, R. \& BIRKS, J. (2004) Donepezil for vascular cognitive impairment. Cochrane Library, issue 4 Oxford: Update Software

MCKEITH, I., DEL SER, T., SPANO, P., et al (2000) Efficacy of rivastigmine in dementia with Lewy bodies: a randomised, double-blind, placebocontrolled international study. Lancet 326, 2031-2036

NATIONAL INSTITUTE FOR CLINICAL EXCELLENCE (2001) Guidance on the Use of Donepezil, Rivastigmine and Galantamine for the Treatment of Alzheimer's Disease. Technology Appraisal Guidance, no. 19. London: NICE.
O'BRIEN, J. \& BALLARD, C. (2001) Drugs forAlzheimer's disease. BMJ, 323,123-24.

SHELDON, T., CULLUM, N., DAWSON, D., et al (2004) What's the evidence that NICE guidance has been implemented? Results from a nationa evaluation using time series analysis, audit of patients' notes, and interviews. BMJ, 329, 999-1004.

WHITE, C. (2004) NICE guidance has failed to end "postcode prescribing". BMJ, 328, 1277

*Christopher O'Loughlin Specialist Registrar, Old Age Psychiatry, Older Peoples' Mental Health Service, Box 311, OPMHS Office, Fulbourn Hospital, Cambridge CB15EF, e-mail: c.oloughlin@btinternet.com, Jon Darley Consultant, Old Age Psychiatry, West Suffolk Hospital, Bury St Edmunds

\section{JASON LUTY, VINCENT PERRY, OKEN UMOH AND DONNA GORMER \\ Validation and development of a self-report outcome measure (MAP-sc) in opiate addiction}

\section{AIMS AND METHOD}

To develop and assess the viability of a self-completion version of the Maudsley Addiction Profile for assessing and monitoring the functioning of opioid-dependent patients. A total of 206 treatmentseeking opioid-dependent patients completed the Maudsley Addiction Profile interview and a selfcompletion version at a single clinic appointment at a substance misuse facility. Scores from both formats were compared using correlation coefficients.

RESULTS
Non-parametric correlation
coefficients between interview and
self-completion version for alcohol,
drug, psychiatric, family and legal
problems correlated in excess of 0.7
for the majority of the 20 items that
were compared.

\author{
CLINICAL IMPLICATIONS \\ A short, self-administered \\ questionnaire version of the \\ Maudsley Addiction Profile is a \\ feasible alternative to the interview \\ for assessing and monitoring \\ treatment of opioid-dependent \\ patients. The questionnaires were \\ usually completed by clients within \\ $15 \mathrm{~min}$. These would be particularly \\ useful in services with very limited \\ staffing time, such as primary care.
}

The policy of the current UK Government towards NHS services includes the statement that:

'There is no place in the modern NHS for the piecemeal adoption of unproven therapies, or for hanging onto outdated, ineffective, treatments' (Department of Health, 1998).

This heralded a new era in which individual health service providers would be expected to monitor and demonstrate their effectiveness. The demonstration of illicit drug use by urine or oral fluid analysis is regarded by many experts as inadequate to determine the effectiveness of the many aspects of care provided by addiction services (McLellan et al, 1985). Although outcome measures such as the Health of the Nation Outcome Scales (HoNOS; Wing et al, 1998) are available for many mental health sub-specialties, there are relatively few brief instruments that addictions services can routinely use to assess patient functioning and outcomes. Such an instrument would be particularly useful given the UK Government's current enthusiasm for expanding the prescribing of injectables, such as diamorphine to intravenous drug users (National Treatment Agency, 2002). There is also increasing pressure to provide rapid access prescribing particularly to patients from the criminal justice system (Best et al, 2002). We therefore have compared a selfcompletion version of the Maudsley Addiction Profile
(MAP) with a short interview to demonstrate that the self-completion version can be used in the routine assessment of those attending substance misuse services.

The standard MAP (Marsden et al, 1998) is a wellvalidated, brief semi-structured interview developed to assess the substance use, risk behaviour, health and social functioning of illicit drug users. It was initially developed as a tool to measure the outcome of substance misuse treatment as a result of the recommendation of the UK Department of Health Task Force on Services for Drug Misusers. Field-testing has shown the instrument to be reliable and valid in people with substance misuse problems.

The MAP requires a health professional to complete the various sections. This takes approximately $12 \mathrm{~min}$. There is no requirement for formal training to use the MAP. Nevertheless, many service providers, particularly those in primary care where doctors or nurses have appointments of less than $10 \mathrm{~min}$, find this time commitment onerous, particularly as part of follow-up appointments.

Many surveys have concluded that self-reporting of drug use is reliable and valid when events are recent and patients do not face negative consequences (McLellan et al, 1985; Mieczkowski, 1990; Poole et al, 1996; Lundy et al, 1997; Darke, 1998). Studies comparing written 